\title{
Myositis and eosinophilia in a patient with sarcoidosis
}

\author{
Z.S. Al-Saffar, C.R. Kelsey, R.P. Kennet and M. Webley \\ Oxford Regional Rheumatic Diseases Research Centre, Stoke Mandeville Hospital, Aylesbury, \\ Bucks HP21 8AL, UK
}

\begin{abstract}
Summary: We present a patient with muscle manifestations of sarcoidosis associated with raised creatine kinase levels, eosinophilia and interstitial lung disease. She had significant improvement on prednisolone $30 \mathrm{mg}$ per day.
\end{abstract}

\section{Introduction}

Sarcoidosis is a multisystem granulomatous disorder of unknown aetiology, commonly involving lymph nodes, lungs, spleen, skin, eyes and parotid glands. The musculoskeletal system is less commonly involved and symptomatic muscle involvement remains rare. We describe a patient who developed progressive muscle involvement associated with eosinophilia many years after having had pulmonary sarcoidosis.

\section{Case report}

A 59 year old West Indian woman presented with a 6 month history of progressive pain and weakness of both legs. Eight years previously she had presented elsewhere with exertional dyspnoea and had been diagnosed as having pulmonary sarcoid. She was treated with steroids for about a year and had recovered. She had mild hypertension treated with nifedipine and a diuretic.

On examination she had generalized muscle weakness, more prominent proximally and in her legs, associated with tenderness of the calf and thigh muscles, but no gross wasting. Muscle strength of quadriceps, plantar flexors and peroneal muscle groups was graded $3 / 5$. Deltoids and biceps were graded $4 / 5$, there was no abnormality of sensation apart from diminished fine touch sensation of her feet. Examination of her chest revealed a few basal crepitations. During her admission her right ankle became swollen and aspirated synovial fluid showed calcium pyrophosphate crystals.

Correspondence: Z.S. Al-Saffar, M.B., Ch.B., M.R.C.P. (U.K.).

Accepted: 28 February 1994
Investigations showed haemoglobin $12.3 \mathrm{~g} / \mathrm{dl}$ and white cell count 3.7 with $52 \%$ neutrophils and $25 \%$ eosinophils (total count $\left.0.19 \times 10^{9} / 1\right)$. The ESR was $68 \mathrm{mmHg}$. Her C-reactive protein level (CRP) was low on admission but rose to $15 \mathrm{mg} / \mathrm{l}$ with ankle synovitis. Routine biochemistry including liver functions tests and calcium were normal. Creatine kinase (CK) levels were elevated at $438 \mathrm{U} /$ 1 (normal range $25-90 \mathrm{U} / \mathrm{l}$ ). Serum angiotensin converting enzyme level was $72 \mu \mathrm{mol} / 1$ (normal 23-67 $\mu \mathrm{mol} / \mathrm{l})$. An electrocardiogram showed Twave inversion in the lateral leads. A chest X-ray showed moderate reticular nodular shadowing in the lower lung fields. Pulmonary function tests confirmed a restrictive defect with low gas transfer. $\mathrm{X}$-ray of the right ankle was normal. A quadriceps biopsy showed focal sarcoid granulomata. There was also marked variation in fibre diameter with small-type grouping and both type I and II fibre atrophy. There was no inflammatory cell infiltrate, and no degenerating or regenerating muscles fibres. A neurophysiological study showed normal sensory and motor nerve conduction. Electromyography of both vastus lateralis muscles showed profuse spontaneous activities consisting of fibrillation potentials and positive sharp waves. The motor unit action potentials (MUAPs) in these muscles and in the right deltoid were abnormally complex with increased numbers of turns and phases. The MUAP amplitude was low, measured at $0.1-0.5 \mathrm{mV}$, but durations were normal. These findings were interpreted as being compatible with active muscle fibre necrosis from an inflammatory primary myopathy.

She was treated with prednisolone $30 \mathrm{mg}$ a day and showed a dramatic improvement in muscle strength. Her ESR, eosinophilia and CK levels all rapidly returned to normal. Her prednisolone dose was gradually reduced to 20 a day over 3 months. Repeat pulmonary function tests have shown some improvement in gas transfer. 


\section{Discussion}

Symptomatic muscle involvement in sarcoidosis remains rare. ${ }^{1,2}$ The most common presentation as seen in our patient is of slowly progressive muscle weakness and wasting closely resembling chronic myositis or muscular dystrophy. ${ }^{3}$ The diagnosis of sarcoidosis in our patient was suggested because of earlier presentation with pulmonary involvement. Though isolated muscle involvement has been reported it normally occurs in the context of a multisystem disorder. ${ }^{4}$ Our patient had evidence of pulmonary fibrosis.

Sarcoid myopathy most typically presents as a slowly progressive symmetrical involvement usually of the proximal limb muscles, though axial, distal and rarely facial and bulbar muscle involvement has also been described. Muscle wasting is usually seen but cases of pseudohypertrophy mimicking musclar dystrophy do occur. ${ }^{3}$ Muscle pain and tenderness is not usually a characteristic feature of the chronic myopathy. It has been suggested that sarcoid myopathy may more commonly present in postmenopausal women. ${ }^{3,5} \mathrm{CK}$ levels are usually moderately elevated.

Other less common types of muscle involvement in sarcoidosis include acute myositis and palpable muscle nodules. Acute myositis has been rarely described, usually in the early stages of sarcoidosis. Muscle pain and tenderness seem to respond well to steroid therapy. ${ }^{6}$ Palpable muscle nodules were the first form of sarcoidosis to be reported but are also very uncommon. It uşually presents with localized pain, stiffness or muscle cramps and may respond to local steroid injection. ${ }^{7}$

Asymptomatic muscle involvement in sarcoidosis is probably much more common. Several series have suggested that about $50 \%$ of patients will show a positive muscle biopsy, which suggests that this may be a useful means of obtaining the histological diagnosis of sarcoid. ${ }^{1,4,8}$ However, there appears to be no correlation between the extent of granuloma formation in the muscle and the clinical findings of muscle weakness. The present of asymptomatic granulomas is well recognized in other tissue affected by sarcoidosis.

Although arthritis is seen in up to $40 \%$ of patients with acute sarcoidosis, it is less common in

\section{References}

1. Silverstein, A. \& Siltzbach, L. Muscle involvement in sarcoidosis. Arch Neurol 1969, 21: 238-241.

2. Myers, G.B., Gottlier, A.M., Mattman, P.E., Eckley, G.M. \& Chason, J.L. Joints and skeletal muscle manifestations in sarcoidosis. Am J Med 1952, 12: 161-169.

3. Douglas, A.C., Macleod, J.G. \& Matthews, J.D. Symptomatic sarcoid of skeletal muscle. J Neurol Neurosurg Psychiatry 1973, 36: 1034-1040. chronic sarcoidosis. Ankles, knees, small joints of the hands and wrists are most commonly involved. $\$ 2$ Hypercalcaemia is a known complication of sar $\leftarrow$ coidosis and can lead to a soft tissue calcification $\overrightarrow{\vec{F}}$ but pyrophosphate arthritis has not previously? been described and indeed, in this case, may be coincidental.

Regarding the investigations, muscle biopsy has a definitive role in the diagnosis of sarcoid myopathy. Muscle infiltrations with non-caseatinges granuloma is the classical finding, and in chronic sarcoid myopathy it causes pressure atrophy, degeneration and fibrosis of the adjacent musclew fibres. ${ }^{3}$ CK levels are expected to be markedlyo increased in acute myositis while modest elevation has been reported in chronic myopathy.$^{10}$ Electro myography, when performed in previous studies, $O$ usually showed a myopathic pattern with occa sional neuropathy.,10 Gallium-67 citrate imaging ${ }^{3}$ has been documented to be useful in the diagnosisc and delineation of sites with involvement of sar-o coid. Increased uptake in the muscle has been reported in muscular sarcoid, which was reversible ${ }_{Z}$ with treatment. ${ }^{11}$

Our patient also exhibited an eosinophilia, and $\overleftrightarrow{\overparen{\Phi}}$ had low or normal CRP levels. Eosinophilia haso only been reported in a few cases of sarcoidosis agd? only in those with severe pulmonary involvemen $12 \overrightarrow{0}$ In our patient there was no other cause for? eosinophilia. The lung involvement was ontyo moderate and the eosinophilia rapidly resolved with steriod therapy. The CRP was normal in our patient except for a transient slight elevation wheng she developed crystal synovitis of her ankle. It has been suggested that the CRP can be a useful test to differentiate between sarcoidosis and othero글 inflammatory granulomatous disease, such as Wegener's or tuberculosis in which the CRP is usually elevated. ${ }^{13}$

As with other types of sarcoidosis, cortico-학 steroids have been recommended for the treatment ${ }_{-}$. of muscle involvement. Most patients, such as the one reported here, have shown a rapid improve- 8 ment on prednisolone at doses of less than $30 \mathrm{mg}$ a day. ${ }^{10}$ However, the response to steroids can be 0 unpredictable. In other series, patients have shown only a poor or partial response, or have relapsed on lower doses.
4. Wallace, S.L., Lattes, R., Malia, J.P. \& Ragon, C. Muscle involvement in Boeck's sarcoid. Ann Intern Med 1958, 48: 497-511.

5. Gardener-Thorpe, C. Muscle weakness due to sarcoid myo- $\stackrel{\oplus}{?}$ pathy. Neurology 1972, 22: 917-928.

6. Alpert, J.N., Groff, A.E., Bastain, F.O. et al. Acute poly- $\frac{0}{0}$ myositis caused by sarcoidosis: report of a case and review of the literature. Mt Sinai J Med 1979, 46: 486-488. 
7. Janssen, M., Dijkmans, B.A.C. \& Eulderink, F. Muscle cramps in the calf as presenting symptoms of sarcoidosis. Ann Rheum Dis 1991, 50: 51-52.

8. Phillips, R.W. \& Phillips, A.M. The diagnosis of Boeck's sarcoid by skeletal muscle biopsy. Arch Int Med 1956, 98: $732-736$.

9. Gumpel, J.M., Johns, C.J. \& Shulman, L.E. The joint disease of sarcoidosis. Ann Rheum Dis 1967, 269: 194-205.

10. Wolfe, S.M., Pinals, R.S., Aelion, J.A. \& Goodman, R.E. Myopathy in sarcoidosis. Semin Arth Rheum 1987, 16: 300-306.
11. Edan, G., Baurguet, P., Delaval, Ph. \& Herry, J.Y. Gallium67 scanning in muscular sarcoidosis. J Nucl Med 1984, 25: 776-778.

12. Pierce, J., Glazer, H., Cook, R., Atkinson, J., Crouch, E. \& Fiedorek, F. Asthma and eosinophilia in a 66 year old woman. Am J Med 1989, 87: 439-444.

13. Peros-Golubicic, T. C-reactive protein in sarcoidosis. Acta Med Iugosl 1991, 45: 169-174. 